University of Nebraska - Lincoln

DigitalCommons@University of Nebraska - Lincoln

Faculty Publications from the Center for Plant

Science Innovation

Plant Science Innovation, Center for

$10-2005$

\title{
Plant organellar protein targeting: A traffic plan still under construction
}

Sally Ann MacKenzie

University of Nebraska-Lincoln, sam795@psu.edu

Follow this and additional works at: https://digitalcommons.unl.edu/plantscifacpub

Part of the Plant Sciences Commons

MacKenzie, Sally Ann, "Plant organellar protein targeting: A traffic plan still under construction" (2005). Faculty Publications from the Center for Plant Science Innovation. 40.

https://digitalcommons.unl.edu/plantscifacpub/40

This Article is brought to you for free and open access by the Plant Science Innovation, Center for at DigitalCommons@University of Nebraska - Lincoln. It has been accepted for inclusion in Faculty Publications from the Center for Plant Science Innovation by an authorized administrator of DigitalCommons@University of Nebraska Lincoln. 


\title{
Plant organellar protein targeting: A traffic plan still under construction
}

\author{
Sally A. Mackenzie \\ Plant Science Initiative, N300 Beadle Center for Genetics Research, \\ University of Nebraska-Lincoln, Lincoln, NE 68588-0660, USA; email smackenzie2@unl.edu
}

\begin{abstract}
It has long been understood that specific features of a protein and its corresponding import apparatus dictate the behavior of mitochondrial proteins in their intracellular targeting behavior. In plants, the process by which proteins are directed to organelles has been influenced uniquely by the introduction to the cell of plastids. Parallel functions carried out within the mitochondrion and plastid permit the sharing of proteins and emergence of mechanisms to facilitate dual-targeting of the nuclear-encoded products to both compartments. These include transcriptional and translational variations, relaxation of translation initiation controls and conditional cellular influences. Details of the dual targeting system are emerging from recent studies, and evidence of variation in protein targeting behavior across plant families and across organisms implies that the system itself is in flux. This trend towards multi-targeting enhances protein versatility across eukaryotes - one means of cellular response to developmental or environmental influence.
\end{abstract}

\section{Introduction}

Proteins possess within their sequence precise information that dictates their cellular location. This well-established biological concept, while essentially true, has undergone considerable revision over the past few years. Cellular protein trafficking is a complex, responsive and rapidly evolving process. For mitochondrial [1] and plastid [2] components, it most often involves specific $\mathrm{N}$-terminal extensions to the nuclear-encoded protein, known as the targeting presequence (mitochondrial) or transit peptide (plastid), interacting with membrane-bound proteins sitting near the surface of the organelle to influence protein import. However, in recent years, the number of proteins found targeted to both mitochondria and plastids has increased significantly, obscuring our understanding of which peptide features dictate organelle targeting specificity and heightening interest in the plant-specific evolutionary processes that have expanded and refined protein targeting capacity. What emerges from recent studies suggests that features of a protein essential for its organellar targeting are often more subtle, readily adapted and susceptible to cellular or developmental influences than was previously thought.

\section{A rationale for protein dual targeting}

Most biologists accept the basic premise first put forward by Margulis [3] that the eukaryotic predecessor to present-day animal and plant cells originated with at least one a-proteobacterial fusion or endosymbiosis event to give rise to the mitochondrion. This process led to a massive, and largely unidirectional, transfer of genetic information to the nucleus, leaving contemporary mitochondrial genomes to encode a very small percentage of the proteins essential for function. Aside from some of the organelle's own translational apparatus, mitochondrially encoded proteins generally range in number from 13 in most animals to around 20-50 in most plants $[4,5]$. Unique to plants, this endosymbiosis was followed by a second event with a cyanobacterial progenitor, supplying photosynthetic capabilities to the cell by the present-day chloroplast. This event was similarly followed by extensive gene transfer to the nucleus, leading to an interesting dilemma for the plant cell. In mitochondrial and chloroplast evolution, a significant portion of the genetic information transferred from these organelles to the nucleus was redundant in function, including the apparatus for their own genome maintenance and expression functions. Yet, there was also considerable genetic information that was highly specialized, required to support the mitochondria-specific functions of oxidative phosphorylation or plastid photosynthesis, for example. The dilemma comes in how to target highly specialized proteins with the necessary precision, while economizing on functionally redundant components.

Evidence from recent studies of plant genomes suggests that inter-organellar gene relocation has occurred in the form of massive and frequent genomic transfers (Box 1). The relocation of organellar genes to the nucleus is followed by acquisition of a functional promoter as well as protein targeting capacity. Little information is available about how the promoter arises, although it seems likely that a newly integrated organellar sequence acquires and adapts the regulatory sequences present at its site of integration. There does exist a growing body of information regarding protein targeting, however. Acquired protein targeting strategies are much more varied than was first thought. 


\section{Box I.The nature of organellar DNA transfers to the nucleus}

The Arabidopsis nuclear genome contains nearly complete copies of the mitochondrial genome on chromosome 2 [4I], and it has been possible to observe chloroplast-to-nucleus DNA transfers in current experiments (reviewed in [42]). These observations imply that direct organelle-to-nucleus DNA transfer is ongoing. However, these direct DNA transfers might not produce functional loci in the nucleus, and studies of active nuclear genes that encode mitochondrial or plastid products do not necessarily support the concept of large multi-gene integrations. For example, genes involved in organellar DNA and RNA maintenance functions appear to be linked within the plant nuclear genome, implying their transfer en masse, but more detailed analysis shows that these clusters intermingle genes that were apparently derived from both mitochondrial and plastid progenitors [10]. In fact, evidence suggests that functional mitochondrial gene transfers to the nucleus often involved RNA intermediates. This observation implies that effective transfer of an organellar gene to the nucleus, at least in recent times following the advent of organelle RNA editing, requires the reverse transcription of organellar mRNA before its integration into the nucleus (reviewed in [43], but see also [5]).

\section{The targeting sequence}

The majority of nuclear-encoded mitochondrial and plastid proteins are translated on cytosolic ribosomes and directed to the appropriate organelle by the N-terminal signal peptide. Plant signal sequences show little conservation and vary greatly in length, ranging from 18 to 107 amino acids for mitochondrial presequences and 13 to 146 for plastid transit peptides. The amino acid compositions of both are similar, highest in hydrophobic (alanine, leucine, phenylalanine or valine) and hydroxylated (serine and threonine) amino acids, as well as positively charged residues (arginine and lysine) [6]. In mitochondrial presequences, the three most abundant amino acids are usually serine, arginine and alanine. The structure of the mitochondrial presequence has the potential to form amphiphilic a-helices that are thought to be important for import, with hydrophobic residues forming interactions with the receptor on the outer mitochondrial membrane. The plastid transit peptide has a slightly less-defined structure, with hydroxylated residues that can form a phosphorylation motif to facilitate interaction with cytosolic proteins for import. In spite of the variability in length and lack of conserved amino acid sequence, single amino acid mutations within the presequence, particularly at hydrophobic residues, can dramatically influence protein import behavior $[7,8]$.

Several years ago, it was shown that an organellar gene introduced to the nuclear genome could acquire targeting information by ectopic DNA recombination with a gene that already contained a presequence [9]. Evidence can be found for this type of presequence exchange in the Arabidopsis genome [10]. However, some organellar genes transferred to the nuclear genome might have already possessed N-terminal features to their proteins that would have facilitated import to the mitochondrion [11]. Previous studies, one using random bacteria-derived sequences [12] and one using computer-randomized sequence [13], showed that a surprising proportion of seemingly random sequences impart organelle protein import activity. This is probably because the most prevalent amino acids in presequences are represented by the most abundant codons in the universal genetic code (serine, arginine and leucine each have six codons, while alanine has four). The least abundant amino acids in targeting presequences (Asp and Glu) are each represented by only two codons. Hence, the features of the presequence essential for targeting might not be as specialized as once suspected.

In plants, protein targeting specificity is also influenced by features of the organellar protein import apparatus. Components of the outer mitochondrial membrane that participate in protein import by interacting directly with the presequence are well conserved in most eukaryotes, but diverge in plants. In particular, the Tom22 subunit, a core member of the outer membrane protein translocation channel, acts as an acidic receptor for the basic targeting presequence of a precursor protein. This acidic cis receptor domain is conserved in fungi, mammals, birds, nematodes and fruit flies but is absent from plants, apparently replaced by truncated Tom 22 homologs that range in size from $\sim 9-11 \mathrm{kDa}$ (reviewed in [14]).

These modifications in plants, although resembling truncations found in some protozoans [15], might have been adapted with introduction of the plastid, an event necessitating more precise protein targeting. The plastid protein import apparatus comprises components distinct from the mitochondrial apparatus [2]. Therefore, within the plant cell, explicit targeting of a protein to one organelle relies on corresponding features contained both within the presequence and the import apparatus.

\section{The nature of dual targeting}

Proteins dual-targeted to both mitochondria and plastids seem to fall into three general categories: proteins involved in DNA and RNA maintenance functions, translation components and proteins associated with cellular defense responses. These include several amino acyl tRNA synthetases, DNA and RNA polymerases, DNA recombination components, a sigma transcription factor $[10,16,17]$, and several pentatricopeptide repeat (PPR) proteins [18], thought to be involved in organellar RNA processing. In Arabidopsis, ascorbate peroxidase, monodehydroascorbate reductase and glutathione reductase gene products, involved in antioxidant defense, also demonstrate mitochondrial and plastid dual targeting [19].

A single protein with the capacity to localize to two distinct organelles must also possess the necessary features to be recognized and properly processed upon import. Until recently, it was unclear how both the mitochondrial and plastid processing peptidases would cleave identical peptides. Appropriately, a zinc metalloprotease (AtZn-MP) that is required to remove the signal peptide from the targeted protein following import shows localization and precursor processing activity in both mitochondria and chloroplasts [20, 21].

The two primary means of permitting dual, mitochondrialplastid targeting of a protein have been classified as 'twin' and 'ambiguous' presequences ([16]; Figure 1). Twin presequences are defined as mitochondrial and chloroplast targeting sequences arranged in tandem. This parallel targeting information is generally included in genes with two transcription start sites, two alternative translation initiation sites, or alternative exon splicing to permit the synthesis of distinct mitochondrial or plastid products. The ambiguous presequence is a sin- 


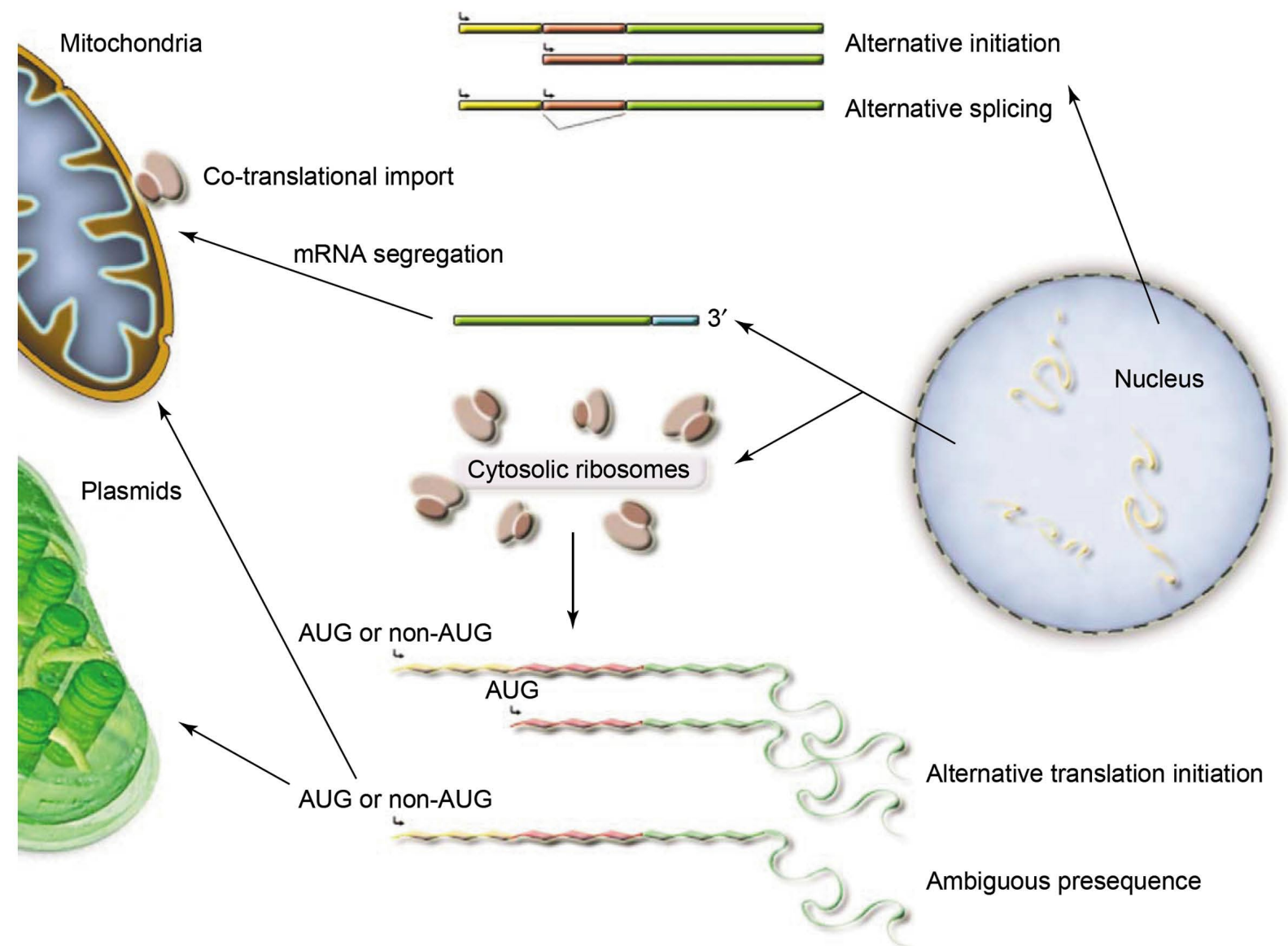

Figure I. Recently emerging details of organellar protein targeting mechanisms. The cartoon depicts some of the basic features of protein targeting that have come to light recently, showing that organellar protein targeting can be influenced at both the transcript (transcription initiation, transcript splicing, transcript features for ribosomal recruitment) and protein (translation initiation, presequence recognition features) level to specify and expand protein targeting capacity. Transcripts are straight and proteins curled figures, with red and yellow depicting plastid and mitochondrial targeting features, respectively, and blue depicting 3 ' transcript features required for mitochondrial ribosomal recruitment. The plastid image in the figure was derived from http://www.daviddarling.info/encyclope$\mathrm{dia} / \mathrm{C} / \mathrm{chloroplasts.html}$

gle targeting sequence recognized by both mitochondria and chloroplasts, with different sites within the presequence interacting with each import apparatus. Interestingly, the first report of protein dual targeting in plants was made with a yeast targeting presequence in transgenic tobacco cells [22]. The fact that a protein from yeast, which contains no plastids, can also target plastids implies that dual targeting can arise fortuitously, with the ability of particular signal peptides to interact with the import apparatus of both organelles.

In the dual-targeted pea glutathione reductase, mutagenesis of the presequence revealed a domain essential for plastid targeting overlapping with a domain essential for mitochondrial targeting $[23,24]$. The means by which a protein acquires dual targeting capacity can vary. In some cases, partial relaxation of translation initiation control has occurred [25]. This relaxation might permit occasional initiation upstream to the recognized initiator MET, resulting in two alternative MET initiator sites. More surprising, however, is that this relaxation can result in the establishment of non-AUG translation initiation sites. This is the case in yeast glycine [26] and alanine [27] tRNA synthetases, to permit cytosolic and mitochondrial dual targeting, and in plant DNA polymerase [13] for mitochondrial-plastid dual targeting. In the organellar DNA polymerase, the upstream initiator CTG appears to be as effective as an ATG in the same position, based on site-directed mutagenesis, and relative mitochondrial versus plastid targeting appears to be modulated developmentally [13].

In the RNA polymerase gene of the moss Physcomitrella patens, two in-frame MET codons are present, with translation initiation at the upstream site conferring dual targeting, whereas initiation at the second confers mitochondrial targeting. When one research group included in their studies the coding sequence from the upstream MET, but omitted the untranslated 5 ' leader sequence, protein targeting was dual [28]. When a second group tested the same locus by incorporating the 5 ' untranslated leader into their reporter gene constructions, initiation occurred at the second MET and targeting was mitochondrial $[29,30]$. These latter results indicate the importance of gene context in selection of the initiator codon and imply that the protein localizes exclusively to mitochondria. The details remain unclear from these studies, however. If the second MET is the only active initiation codon, why then would other plant species have conserved the two MET translation initiator codons [31]? Moreover, if this protein is exclusively mitochondrial in targeting, it is curious that mutation of the homologous RNA polymerase gene in Arabidopsis results in a plastid phenotype. Perhaps a more likely explanation is that the first MET is conditionally active. 
Box 2. Technical advances allow more detailed resolution of protein targeting in plant cells.

Significant advancement in our understanding of plant protein targeting determinants has come with the introduction of more informative in vivo experimental procedures. One strategy uses fluorescent protein reporter genes in fusion gene constructions with signal peptide sequences and laser confocal microscopic analysis of transient and stable transformants. This approach dramatically extends our ability to reveal the effects of detailed sitedirected modifications of targeting information on the in vivo cellular behavior of the green fluorescent protein (GFP) and to discover protein targeting information located within sequences residing upstream of the annotated gene. What has emerged from these experiments suggests a trend in post-endosymbiotic gene evolution towards protein versatility by multi-targeting. However, given the responsiveness of protein targeting behavior to cellular conditions [34], such targeting assay procedures cannot substitute for high-resolution subcellular fractionation to localize proteins accurately. Figure I shows examples of plant organellar protein targeting results with a transient expression assay and biolistic particle bombardment. The enhanced GFP gene has been fused to plastid [Rubisco small subunit, panel (a)], mitochondrial [FI-ATPase $\gamma$ subunit, panel (b)] and dual targeting [DNA polymerase Atl g50840, panel (c)] signal peptides, and expressed under the control of the CaMV 35 S promoter. Gene constructions were introduced to young Arabidopsis leaf cells and assayed for expression by laser confocal microscopy $24 \mathrm{~h}$ following bombardment. Transiently expressing cells are epidermal, so that unaffected plastids in underlying cells autofluoresce red. Images were taken, with permission, from Christensen et al. [13]. Bars, I7 $\mu \mathrm{m}$.
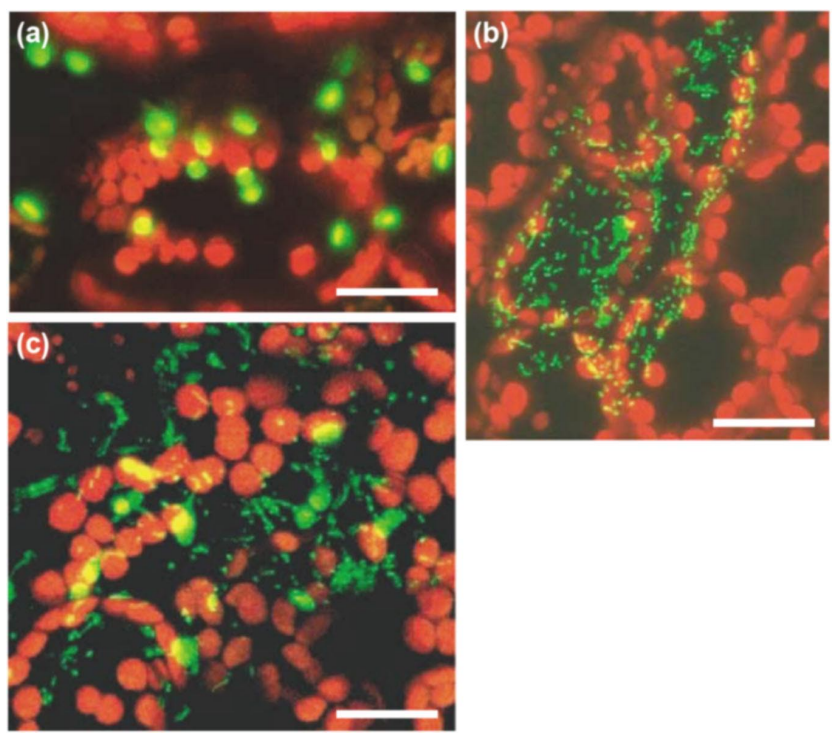

Figure I.

These seemingly contradictory experimental outcomes point to the need to integrate comparative genomic and mutational analysis with protein localization assays in these types of investigations. Important technical advances have opened the way for more refined in vivo analyses in plants (Box 2). Likewise, cellular and developmental factors can influence the activity of one translation initiation site over another. Some reports have described dual targeting in a GFP reporter assay as a non-simultaneous process, with predominant localization to mitochondria observed in one experiment, and to plastids when the experiment is repeated [32]. These sorts of experimental ambiguities are not uncommon and can reflect genuine fluctuations in the system.

\section{Protein targeting is a dynamic and responsive system}

Although protein dual targeting is commonly observed for proteins that function within the mitochondrion and chloroplast, proteins are also dual targeted to the mitochondrion and peroxisome, and the nucleus or the cytosol. Biological adaptations underpinning the evolution of dual targeting are now beginning to emerge. One intriguing example in animals involves the cellular contrasts between herbivory and carnivory in particular animal lineages. The metabolic enzyme alanine: glyoxylate aminotransferase (AGT) participates in the detoxification of glyoxylate to glycine, preventing the accumulation of oxalate. In herbivores, glycolate is thought to accumulate in the peroxisome, and the concentration of AGT would be expected to be highest at this site of action. Peroxisomal protein targeting involves a C-terminal peptide. However, in the carnivore, hydroxyproline is speculated to be the primary glyoxylate precursor, with its conversion occurring in the mitochondrion. The mitochondrial signal peptide is N-terminal. A recent study of the cellular localization of AGT in carnivores and herbivores provides evidence to suggest that differential skewing of this dual-targeted enzyme appears to correlate with diet transitions in the evolution of the order Carnivora [33]. At least part of this selection appears to have occurred by amino acid substitutions within the N-terminal mitochondrial presequence to enhance or reduce the efficiency of mitochondrial targeting relative to peroxisomal targeting.

The targeting properties of a protein are dramatically influenced by cellular conditions (reviewed in [34]). In the case of catalase A (Cta1p) in yeast, an important $\mathrm{H}_{2} \mathrm{O}_{2}$ scavenger, the protein contains peroxisome targeting signals at both the carboxy end and within the N-terminal third of the protein, and no clearly recognizable mitochondrial presequence. Catalase A is efficiently targeted to the peroxisome when the cells are grown on oleate. However, in spite of the apparent absence of a presequence, the protein is localized to the mitochondrial matrix when the cells are enhanced in respiration by growth on the non-fermentable carbon source raffinose [35]. These cellular-responsive dual distributions can be highly disparate in their relative levels, a phenomenon termed eclipsed distributions; this is the case for aconitase in the cytosol and the mitochondrion [36].

Although environmental and metabolic influences on plant protein localization are not yet well characterized, one key regulator is clearly light. For example, the nucleocytoplasmic partitioning of photoreceptors is influenced by light. Subcellular localization of phytochromes A, B, C, D and E, and the ability to form speckle structures following nuclear import, demonstrates a direct response to light and a diurnal rhythm [37].

Plant cytoplasmic features, not yet identified, have also been found to influence the nuclear-encoded portion of the mitochondrial proteome [38]. Mitochondrial protein analysis comparing two isonuclear lines, one containing the male sterility-conferring T cytoplasm and the second a normal (NA) cytoplasm, revealed at least a threefold difference between the lines in accumulation of $13 \%$ of the nuclear-encoded mitochondrial proteins identified. 


\section{Identifying novel cellular components of protein targeting}

The early protein targeting research was largely focused on understanding key features of the presequence and import machinery, but protein targeting mechanisms and influences beyond these also exist (Box 3 ).

The effects of additional trans-acting cellular components on protein targeting properties can be detected by using various in vitro transcription-translation systems. The nuclear-encoded mitochondrial transcription factor Mtf1p of yeast is imported to mitochondria by virtue of a cleavable presequence. When translated in the rabbit reticulocyte lysate system, the full-length Mtf1p translation product is competent for import to mitochondria. However, when the wheat germ extract translation system is used, only the Mtflp product lacking the $\mathrm{N}$ terminal targeting presequence is effectively translocated into mitochondria. Even more surprising was the observation that this translation product appears to be targeted independently of the cleavable presequence, mitochondrial membrane potential or ATP that are normally required for transport by previously defined pathways [39]. Differences in import and dual targeting competence were also found for plant organellar proteins when they were subjected to the two in vitro translation systems [40]. The simplest explanation for these unexpected results is that the two in vitro translation systems contain different sets of chaperones, thus influencing the import-competence state of the translation product. One can envision similar variation across species and/or cell types to confer conditional targeting properties to some types of proteins.

\section{Concluding remarks}

Recent advances in our understanding of protein targeting and import suggest that the early evolution of protein trafficking to mitochondria and chloroplasts involved at least two routes. The refinement of the $\mathrm{N}$-terminal signal peptide might have capitalized on $\mathrm{N}$-terminal amphiphilic properties already common to some proportion of gene products derived from the endosymbiont. The ability of genes, once in the nucleus, to share these targeting features through recombination was likely key to this adaptive process. Likewise, a partial relaxation in control of translation initiation has facilitated acquisition of upstream presequences, suggested by present-day nuclear-encoded organellar proteins initiating at non-ATG codons. This relaxation process probably also accounts for the emergence of dual targeting.

The targeting behavior of proteins appears to rely on at least three newly recognized components (Figure 1). The 5' untranslated leader sequence of a gene is an important influence on translation initiation of dual-targeted proteins. Trans-acting cellular components, some remaining to be identified, are also crucial, presumably accounting for developmental differences in protein targeting behavior and those observed with alternative in vitro translation systems. Even features of the passenger protein itself, including C-terminal targeting information and the influence of conditional protein folding properties, are proving to be important aspects of targeting and import. As our appreciation of the intricacy of protein targeting and the extent of multi-targeting in plants increases, the unambiguous designation of a legitimate component of the mitochondrial proteome grows more challenging every day.

\section{Acknowledgments}

I wish to dedicate this review to the memory of Lee McIntosh; we had hoped to write this review together had time allowed. I extend my apologies to colleagues whose work was not cited owing to space limitations. I thank Alan Christensen for helpful discussions about the manuscript, and Saleem Mohammed for invaluable assistance with figures. Research in my laboratory is supported by grants from National Science Foundation, Department of Energy and the Biotechnology Research Development Corporation.

\section{Box 3. Beyond the targeting signal}

Presequences have co-evolved with the proteins they target. Consequently, varying the passenger protein can influence, sometimes dramatically, the efficiency of protein targeting and import. This was shown for an ATP synthase component in plants [44]. In fact, protein folding during and subsequent to import can also influence targeting behavior. In yeast, the fumarase (FUMI) gene product, encoding a mitochondrial presequence, is distributed to both the mitochondrion and the cytosol. Once imported and processed within the mitochondrion, the protein relies on particular folding to facilitate its re-export to the cytosol through the translocation pore [45].

In some cases, $\mathrm{N}$-terminal sequence features make it impossible to predict targeting behavior of a protein. Several plant organellar proteins appear to initiate translation at non-ATG start codons. This has been suggested for genes encoding RNA polymerase [3I], DNA gyrase [13] and a DNA helicase [13]. In organisms other than plants, non-canonical, $\mathrm{N}$-terminal mitochondrial targeting signals have been identified. This is the case for a WD repeat protein found in the mitochondria of trypanosomes [46]. The protein is targeted by a II 5 amino acid peptide at the $\mathrm{N}$-terminus of the protein that lacks the amphipathic a-helical structure characteristic of targeting presequences. Moreover, a yeast mitochondrial helicase and a dual nuclear-mitochondrial Caenorhabditis ele- gans host cell factor contain mitochondrial targeting information at the carboxy end of the proteins $[47,48]$. The computer-based algorithms for protein targeting prediction currently available are simply not adequate to address this degree of variation in protein targeting determinants, and new algorithms are going to be needed to assess the extent to which these variations occur.

Some organellar targeted proteins contain no targeting information within the protein. These proteins are imported co-translationally at the surface of the mitochondrion. In yeast, several nuclear gene transcripts that encode mitochondrial proteins segregate to the organellar outer membrane surface via mitochondrially bound polysomes. In fact, mammalian mitochondria appear to possess specific receptors for binding ribosomes [49]. Genes of ancient bacterial origin comprise a significant proportion of these mitochondrion-associated mRNAs, while genes of eukaryotic origin that encode organellar proteins generally contain presequences and seem to be primarily translated on cytoplasmic polysomes [50]. The ribosomal recruitment of mRNAs requires specific features of the $3^{\prime}$ untranslated region of the transcripts [5I]. mRNA segregation as an alternative, co-translational route for organellar protein import has only been described in mammalian and fungal cells to date, but it seems likely that such a system will be found in plant cells as well. 


\section{References}

1 C. M. Koehler, New developments in mitochondrial assembly, Annu. Rev. Cell Dev. Biol. 20 (2004), pp. 309-335.

2 P. Jarvis and C. Robinson, Mechanisms of protein import and routing in chloroplasts, Curr. Biol. 14 (2004), pp. R1064-R1077.

3 L. Margulis, Origin of Eukaryotic Cells, Yale University Press (1970).

4 C. E. Bullerwell and M. W. Gray, Evolution of the mitochondrial genome: protist connections to animals, fungi and plants, Curr. Opin. Microbiol. 7 (2004), pp. 528-534.

5 J. N. Timmis et al., Endosymbiotic gene transfer: organelle genomes forge eukaryotic chromosomes, Nat. Rev. Genet. 5 (2004), pp. 123-134.

6 X-P. Zhang and E. Glaser, Interaction of plant mitochondrial and chloroplast signal peptides with the Hsp70 molecular chaperone, Trends Plant Sci. 7 (2002), pp. 14-21.

7 G. Duby et al., Hydrophobic residues within the predicted N-terminal amphiphilic alpha-helix of a plant mitochondrial targeting presequence play a major role in in vivo import, Plant J. 27 (2001), pp. 539-549.

8 F. Ambard-Bretteville et al., Discrete mutations in the presequence of potato formate dehydrogenase inhibit the in vivo targeting of GFP fusions into mitochondria, Biochem. Biophys. Res. Commun. 311 (2003), pp. 966-971.

9 K. Kadowaki et al., Targeting presequence acquisition after mitochondrial gene transfer to the nucleus occurs by duplication of existing targeting signals, EMBO J. 15 (1996), pp. 6652-6661.

10 A. Elo et al., Nuclear genes encoding mitochondrial proteins for DNA and RNA metabolism are clustered in the Arabidopsis genome, Plant Cell 15 (2003), pp. 1619-1631.

11 R. Lucattini et al., Bacterial proteins predisposed for targeting to mitochondria, Mol. Biol. Evol. 21 (2004), pp. 652-658.

12 A. Baker and G. Schatz, Sequences from a prokaryotic genome or the mouse dihydrofolate reductase gene can restore the import of a truncated precursor protein into yeast mitochondria, Proc. Natl. Acad. Sci. USA 84 (1987), pp. 3117-3121.

13 Christensen, A. C. et al. Dual-domain, dual-targeting organellar protein presequences within higher plants may utilize non-AUG start codons. Plant Cell (in press).

14 D. Macasev et al., How do plant mitochondria avoid importing chloroplast proteins: Components of the import apparatus Tom20 and Tom22 from Arabidopsis differ from their fungal counterparts, Plant Physiol. 123 (2000), pp. 811-816.

15 D. Macasev et al., Tom22', an 8-kDa trans-site receptor in plants and protozoans, is a conserved feature of the TOM complex that appeared early in the evolution of eukaryotes, Mol. Biol. Evol. 21 (2004), pp. 1557-1564.

16 N. Peeters and I. Small, Dual targeting to mitochondria and chloroplasts, Biochim. Biophys. Acta 1541 (2001), pp. 54-63.

17 M. C. Silva-Filho, One ticket for multiple destinations: dual targeting of proteins to distinct subcellular locations, Curr. Opin. Plant Biol. 6 (2003), pp. 589-595.

18 C. Lurin et al., Genome-wide analysis of Arabidopsis pentatricopeptide repeat proteins reveals their essential role in organelle biogenesis, Plant Cell 16 (2004), pp. 2089-2103.
19 O. Chew et al., Molecular definition of the ascorbate-glutathione cycle in Arabidopsis mitochondria reveals dual targeting of antioxidant defenses in plants, J. Biol. Chem. 278 (2003), pp. 46869-46877.

20 S. Bhushan et al., Dual targeting and function of a protease in mitochondria and chloroplasts, EMBO Rep. 4 (2003), pp. 1073-1078.

21 C. Rudhe et al., Processing of the dual targeted precursor protein of glutathione reductase in mitochondria and chloroplasts, J. Mol. Biol. 343 (2004), pp. 639-647.

22 J. Huang et al., A yeast mitochondrial leader peptide functions in vivo as a dual targeting signal for both chloroplasts and mitochondria, Plant Cell 2 (1990), pp. 1249-1260.

23 C. Rudhe et al., N-terminal domain of the dual-targeted pea glutathione reductase signal peptide controls organellar targeting efficiency, J. Mol. Biol. 324 (2002), pp. 577-585.

$24 \mathrm{O}$. Chew et al., Characterization of the targeting signal of dual-targeted pea glutathione reductase, Plant Mol. Biol. 53 (2003), pp. 341-356.

25 N. N. Hashimoto et al., Translation initiation at non-AUG codons mediated by weakened association of eukaryotic initiation factor (eIF) 2 subunits, Biochem. J. 367 (2002), pp. 359-368.

26 K. J. Chang and C. C. Wang, Translation initiation from a naturally occurring non-AUG codon in Saccharomyces cerevisiae, J. Biol. Chem. 279 (2004), pp. 13778-13785.

27 H. L. Tang et al., Translation of a yeast mitochondrial tRNA synthetase initiated at redundant non-AUG codons, J. Biol. Chem. 279 (2004), pp. 49656-49663.

28 U. Richter et al., Two RpoT genes of Physcomitrella patens encode phage-type RNA polymerases with dual targeting to mitochondria and plastids, Gene 290 (2002), pp. 95-105.

29 Y. Kabeya et al., Identification and characterization of two phage-type RNA polymerase cDNAs in the moss Physcomitrella patents: Implication of recent evolution of nuclear encoded RNA polymerase of plastids in plants, Plant Cell Physiol. 43 (2002), pp. 245-255.

30 Y. Kabeya and N. Sato, Unique translation initiation at the second AUG codon determines mitochondrial localization of the phage-type RNA polymerases in the moss Physcomitrella patens, Plant Physiol. 138 (2005), pp. 369-382.

31 B. Hedtke et al., Six active phage-type RNA polymerase genes in Nicotiana tabacum, Plant J. 30 (2002), pp. 625-637.

32 T. A. Beardslee et al., A nucleus-encoded maize protein with sigma factor activity accumulates in mitochondria and chloroplasts, Plant J. 31 (2002), pp. 199-209.

33 G. M. Birdsey et al., Differential enzyme targeting as an evolutionary adaptation to herbivory in Carnivora, Mol. Biol. Evol. 21 (2004), pp. 632-646.

34 S. Karniely and O. Pines, Single translation-dual destination: mechanisms of dual protein targeting in eukaryotes, EMBO Rep. 6 (2005), pp. 420-425.

35 V. Y. Petrova et al., Dual targeting of yeast catalase A to peroxisomes and mitochondria, Biochem. J. 380 (2004), pp. 393-400.

36 Regev-Rudzki, N. et al. Yeast aconitase in two locations and two metabolic pathways: Seeing small amounts is believing. Mol. Biol. Cell (in press). 
37 S. Kircher et al., Nucleocytoplasmic partitioning of the plant photoreceptors phytochrome A, B, C, D, and E is regulated differentially by light and exhibits a diurnal rhythm, Plant Cell 14 (2002), pp. 1541-1555.

38 F. Hochholdinger et al., Cytoplasmic regulation of the accumulation of nuclear-encoded proteins in the mitochondrial proteome of maize, Plant J. 37 (2004), pp. 199-208.

39 T. K. Biswas and G. S. Getz, Requirement of different mitochondrial targeting sequences of the yeast mitochondrial transcription factor Mtf1 $\mathrm{p}$ when synthesized in alternative translation systems, Biochem. J. 383 (2004), pp. 383-391.

$40 \mathrm{P}$. Dessi et al., Investigations on the in vitro import ability of mitochondrial precursor proteins synthesized in wheat germ transcription-translation extract, Plant Mol. Biol. 52 (2003), pp. 259-271.

41 R. M. Stupar et al., Complex mtDNA constitutes an approximate $620-\mathrm{kb}$ insertion on Arabidopsis thaliana chromosome 2: implication of potential sequencing errors caused by large-unit repeats, Proc. Natl. Acad. Sci. USA 98 (2001), pp. 5099-5103.

42 W. Martin, Gene transfer from organelles to the nucleus: Frequent and in big chunks, Proc. Natl. Acad. Sci. USA 100 (2003), pp. 8612-8614.

43 K. L. Adams and J. D. Palmer, Evolution of mitochondrial gene content: gene loss and transfer to the nucleus, Mol. Phylogenet. Evol. 29 (2003), pp. 380-395.

$44 \mathrm{M}-\mathrm{N}$. Lee and J. Whelan, Identification of signals required for import of the soybean $\mathrm{F}_{\mathrm{A}} \mathrm{d}$ subunit of ATP synthase into mitochondria, Plant Mol. Biol. 54 (2004), pp. 193-203.
45 E. Sass et al., Folding of fumarase during mitochondrial import determines its dual targeting in yeast, J. Biol. Chem. 278 (2003), pp. 45109-45116.

46 E. V. Bromley et al., The amino terminal domain of a novel WD repeat protein from Trypanosoma cruzi contains a noncanonical mitochondrial targeting signal, Int. J. Parasitol. 34 (2004), pp. 63-71.

47 C. Lee et al., The DNA helicase, Hmi1p, is transported into mitochondria by a C-terminal cleavable targeting signal, J. Biol. Chem. 274 (1999), pp. 20937-20942.

48 A. Izeta et al., A C-terminal targeting signal controls differential compartmentalization of Caenorhabditis elegans host cell factor (HCF) to the nucleus or mitochondria, Eur. J. Cell Biol. 82 (2003), pp. 495-504.

49 J. A. MacKenzie and R. M. Payne, Ribosomes specifically bind to mammalian mitochondria via protease-sensitive proteins on the outer membrane, J. Biol. Chem. 279 (2004), pp. 9803-9810.

50 P. Marc et al., Genome-wide analysis of mRNAs targeted to yeast mitochondria, EMBO Rep. 3 (2002), pp. 159-164.

51 M. Corral-Debrinski et al., In yeast, the 3' untranslated region or the presequence of ATM1 is required for the exclusive localization of its mRNA to the vicinity of mitochondria, Mol. Cell. Biol. 20 (2000), pp. 7881-7892. 